\title{
Paclitaxel and platinum-based chemotherapy results in transient dyslipidemia in cancer patients
}

\author{
GUOPING WANG, CHAO SU and TAO YIN \\ State Key Laboratory of Biotherapy and Cancer Center, West China Hospital, Sichuan University, \\ and Collaborative Innovation Center of Biotherapy, Chengdu, Sichuan 610041, P.R. China
}

Received January 30, 2016; Accepted August 19, 2016

DOI: $10.3892 /$ mco.2016.1107

\begin{abstract}
Paclitaxel and cisplatin (TP) are common chemotherapeutic agents extensively used for treating lung and esophageal cancers. The present study reported three patients with transient hypertriglyceridemia (HTG) following TP chemotherapy. Serum triglyceride (TG) levels returned to baseline at chemotherapy intermission. No patient had any history of HTG or any evidence of pancreatitis or other complications of HTG. No regular elevation of any other serum lipids, including cholesterol, high-density lipoprotein and low-density lipoprotein, was observed. However, treatment of mice with TP decreased TG and slightly increased cholesterol. The findings of the present study suggested that TP chemotherapy results in transient dyslipidemia, and physicians must monitor TG levels during chemotherapy to avoid TG-associated complications.
\end{abstract}

\section{Introduction}

Cancer patients frequently present with metabolic disorders, including cachexia, with hallmarks of atrophy of fat (1). Apart from the systemic stimulatory effects of obesity on tumor growth, adipocytes directly influence tumor behavior. The omentum is primarily composed of adipocytes. Of note, intra-abdominal tumors have a predilection for omentum metastasis (2). Nieman et al (3) demonstrated that human omental adipocytes provide energy for rapid tumor growth and promote homing, migration and invasion of ovarian cancer cells. In this process, adipokines, including interleukin (IL)- 8 serve important roles. In obese patients, adipose stromal cells (ASCs) are expanded and subsequently migrate from the endogenous

Correspondence to: Dr Tao Yin, State Key Laboratory of Biotherapy and Cancer Center, West China Hospital, Sichuan University, and Collaborative Innovation Center of Biotherapy, 17 People's South Road Section Three, Chengdu, Sichuan 610041, P.R. China

E-mail: yintao03073@163.com

Key words: hypertriglyceridemia, chemotherapy, paclitaxel, cisplatin white adipose tissue to tumors, where they are incorporated into blood vessels as pericytes (4). Further evidence has shown that cancer-associated adipocytes modify the cancer cell phenotype leading to a more aggressive behavior (5). Of note, lipid oxidative metabolic programs are essential for regulatory T cells (6) and M2-type macrophages (7), which are classical immune suppressive subsets facilitating tumor growth. Apolipoprotein E (ApoE), targeted by a set of microRNAs, has been demonstrated to be an antiangiogenic and metastasis-suppressive factor in melanoma (8). ApoA-I binding protein accelerates cholesterol efflux from endothelial cells and regulates angiogenesis (9). However, whether chemotherapy influences lipid levels remains to be fully elucidated.

Hypertriglyceridemia (HTG) can occur in patients with cardiovascular diseases, diabetes mellitus, obesity, metabolic syndrome or chronic renal failure (10). Pregnancy, alcohol abuse and certain medications have also been reported to increase the TG concentration $(11,12)$. Elevated plasma levels of TG increase the risk of cardiovascular diseases, diabetes, obesity and insulin resistance $(13,14)$. Furthermore, elevated serum TG levels are associated with hepatosplenomegaly, xanthomas, neuropathy, lipemia retinalis and even hyperlipemic abdominal crisis $(15,16)$. Of note, HTG is a well-recognized cause of acute pancreatitis $(17,18)$, which has a high mortality rate (19).

At our clinic, numerous patients who experienced transient HTG after treatment with paclitaxel and cisplatin (TP) chemotherapy were encountered. The present study reported on three patients with transient HTG after receiving TP chemotherapy. TG returned to baseline at chemotherapy intermission. No patients had any history of HTG or exhibited any evidence of pancreatitis or other complications of HTG. No regular elevation of any other serum lipids, including cholesterol, high-density lipoprotein (HDL) and low-density lipoprotein (LDL), was observed. However, treatment of mice with TP decreased serum TG and slightly increased cholesterol. The present study suggested that, to avoid TG-associated complications, clinicians must monitor TG levels during chemotherapy.

\section{Methods}

Clinical cases. Retrospective data from the electronic medical records of West China Hospital (Chengdu, China) were collected. All three patients selected received chemotherapy at the Cancer Center of West China Hospital. 
Animal study. C57BL/6 and BALB/c mice (6-8 weeks old) were purchased from Beijing HFK Bioscience Co., Ltd. (Beijing, China). Mice were maintained under pathogen-free conditions with individual ventilation (temperature: $21-27^{\circ} \mathrm{C}$; humidity: 40-60\%), with a light/dark cycle of $12 \mathrm{~h}$, and ad libitum access to food and water. The study was approved by the ethics committee of Sichuan University (Chengdu, China). All animal experiments were performed according to protocols approved by the Institutional Animal Care and Use Committee of Sichuan University. A total of $10 \mathrm{mg} / \mathrm{kg}$ paclitaxel was administered on day 1 , and $5 \mathrm{mg} / \mathrm{kg}$ cisplatin was administered on days 1,2 and 3. Chemotherapeutic drugs were administered intravenously to female BALB/c mice $(\mathrm{n}=3$ for each group) and intraperitoneally to C57BL/6 mice. Mice in the control group were administered normal saline. At day 4, blood was collected, the mice were sacrificed and levels of the serum lipids were examined at Chengdu GLP Center (Chengdu, China). The chemotherapeutic agents, paclitaxel and cisplatinum, were obtained from West China Hospital (Chengdu, China).

Statistical analysis. Statistical analysis was performed using Student's t-test between two groups. $\mathrm{P}<0.05$ was considered to indicate a statistically significant difference.

\section{Results}

TP chemotherapy induces serum TG fluctuation in cancer patients. The following three noteworthy cases were encountered at West China Hospital (Chengdu, China). A 47-year-old female patient (patient 1) was diagnosed with lung squamous carcinoma. Since the tumors had invaded the laryngeal recurrent nerve, surgery was not possible and chemotherapy was applied. TP was administered six times and the patient received concurrent radiotherapy. An elevation of serum TG levels was observed each time following chemotherapy. Of note, the HTG was transient and TG levels dropped to normal levels prior to the next cycle of chemotherapy (Fig. 1).

A 66-year-old female patient (patient 2) who had a 3-month history of progressive dysphagia was referred to West China Hospital and diagnosed with esophageal squamous carcinoma. Upon admission to the West China Hospital, the patient had normal serum TG levels. The patient received four cycles of TP chemotherapy and radiotherapy after the third cycle. The patient had no history of dyslipidemia; however, she experienced HTG following every administration of TP (Fig. 1).

A 57-year-old male patient (patient 3) with a history of synovial carcinoma in the left lower extremity was admitted to West China Hospital due to the recurrence and lung metastasis of synovial carcinoma. The patient had undergone excision of synovial carcinoma 13 years previously and upper left pulmonary wedge resection 7 months previously. Thereafter, the patient received 5 cycles of TP chemotherapy. He exhibited HTG after each treatment with TP chemotherapy, which decreased in the subsequent interval (Fig. 1).

Triglyceride disorders are not accompanied by any other lipid disorders. Since chemotherapy with TP influenced serum TG levels, further review of the patients' medical records was performed to determine whether dyslipidemia was also present for cholesterol, LDL and HDL. The trend of these
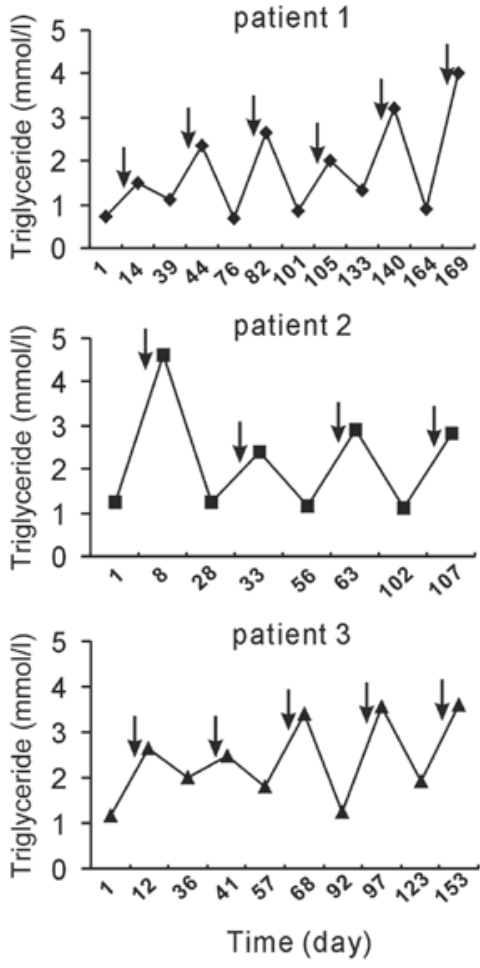

Figure 1. Chemotherapy with TP induced a fluctuation in serum triglyceride levels. Arrows indicate the time-points of TP chemotherapy administration. TP chemotherapy induced transient hyperglyceridemia. Normal range of serum TG: 0.29-1.83 mmol/1. Patients: 1, lung squamous carcinoma; 2 , esophageal squamous carcinoma; 3 , synovial carcimoma with lung metastasis. TP, paclitaxel and cisplatin.

lipids observed following chemotherapy was not similar to that of serum TG (Fig. 2).

Influence of TP chemotherapy on liver and kidney function. Furthermore, serum levels of albumin, ALT and AST after chemotherapy were compared at various time-points. It was revealed that chemotherapy induced a slight elevation of ALT and/or AST in each of the three patients; however, levels almost remained in the normal range. Unlike ALT and AST, albumin levels did not fluctuate following chemotherapy (Fig. 3). In addition, no regular elevation of creatinine and urea was observed following TP chemotherapy (Fig. 4).

Animal model of TP chemotherapy-induced lipid disorder. To investigate whether TP chemotherapy causes a lipid disorder in experimental animals, BALB/c mice were administered intravenously with chemotherapeutic drugs. To mimic the clinical settings, the animals were administered paclitaxel $(10 \mathrm{mg} / \mathrm{kg}$ body weight) at day 1 and cisplatin $(5 \mathrm{mg} / \mathrm{kg}$ body weight) at days 1,2 and 3. At day 4, serum from the mice was obtained to evaluate the TG and cholesterol levels. TG was revealed to be significantly decreased and cholesterol levels were increased (Fig. 5). Furthermore, the same experiment was performed on C57BL/6 mice with intraperitoneal injection of the drugs. In C57BL/6 mice, the same trend was observed as in BALB/c mice, while not reaching statistical significance (Fig. 5). These results suggested that TP chemotherapy caused lipid disorders in mice, while they were not consistent with those in human subjects. 

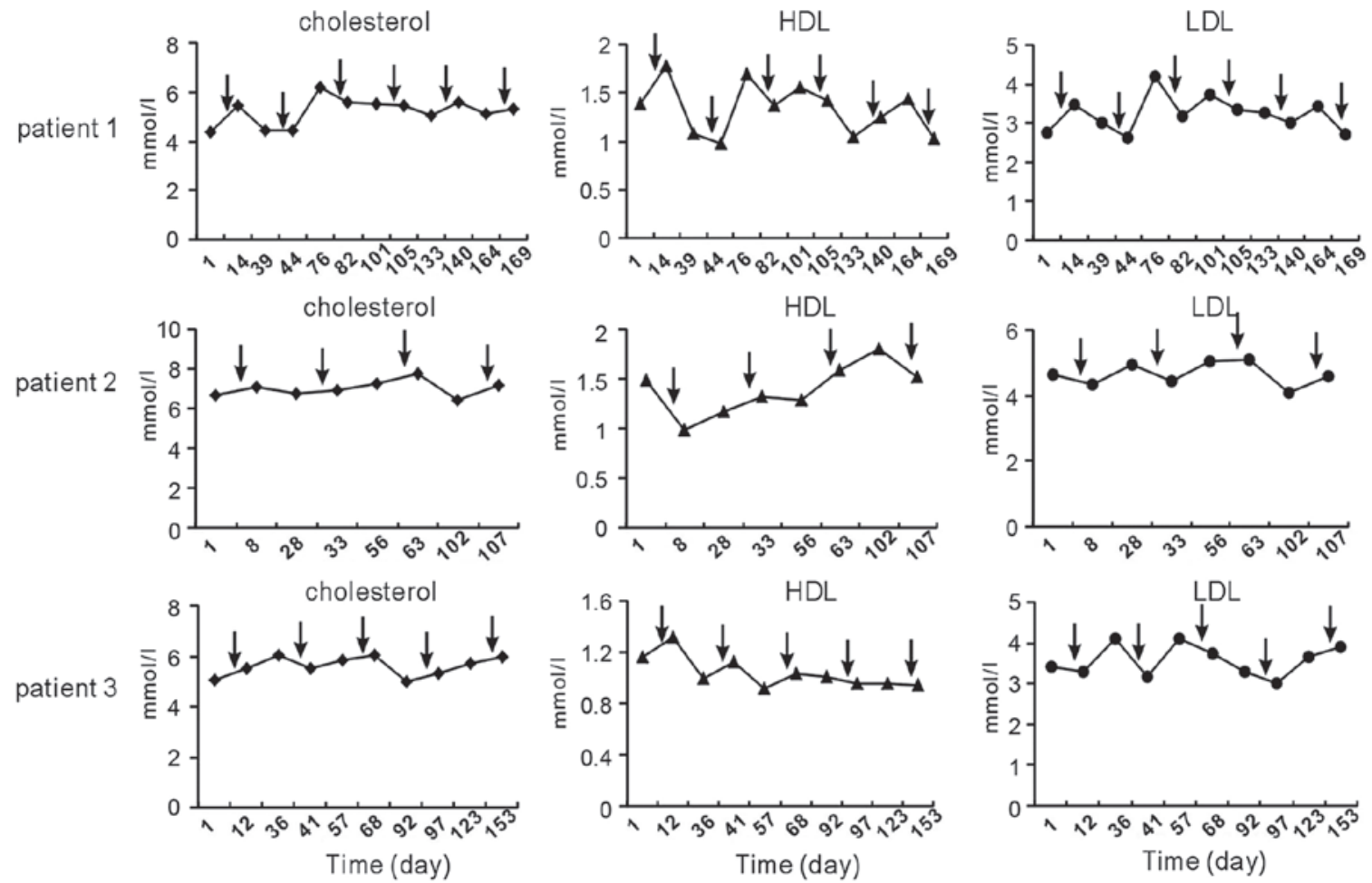

Figure 2. Serum cholesterol, HDL and LDL levels before and after TP chemotherapy in patients 1-3. Arrows indicate the time-points of TP chemotherapy. The fluctuation trend of cholesterol, HDL and LDL after chemotherapy was dissimilar to that of triglycerides. Normal range: cholesterol, 2.8-5.7 mmol/1; HDL $>0.9 \mathrm{mmol} / 1$; LDL $<4.0 \mathrm{mmol} / 1$. HDL, high-density lipoprotein; LDL, low-density lipoprotein; TP, paclitaxel and cisplatin.
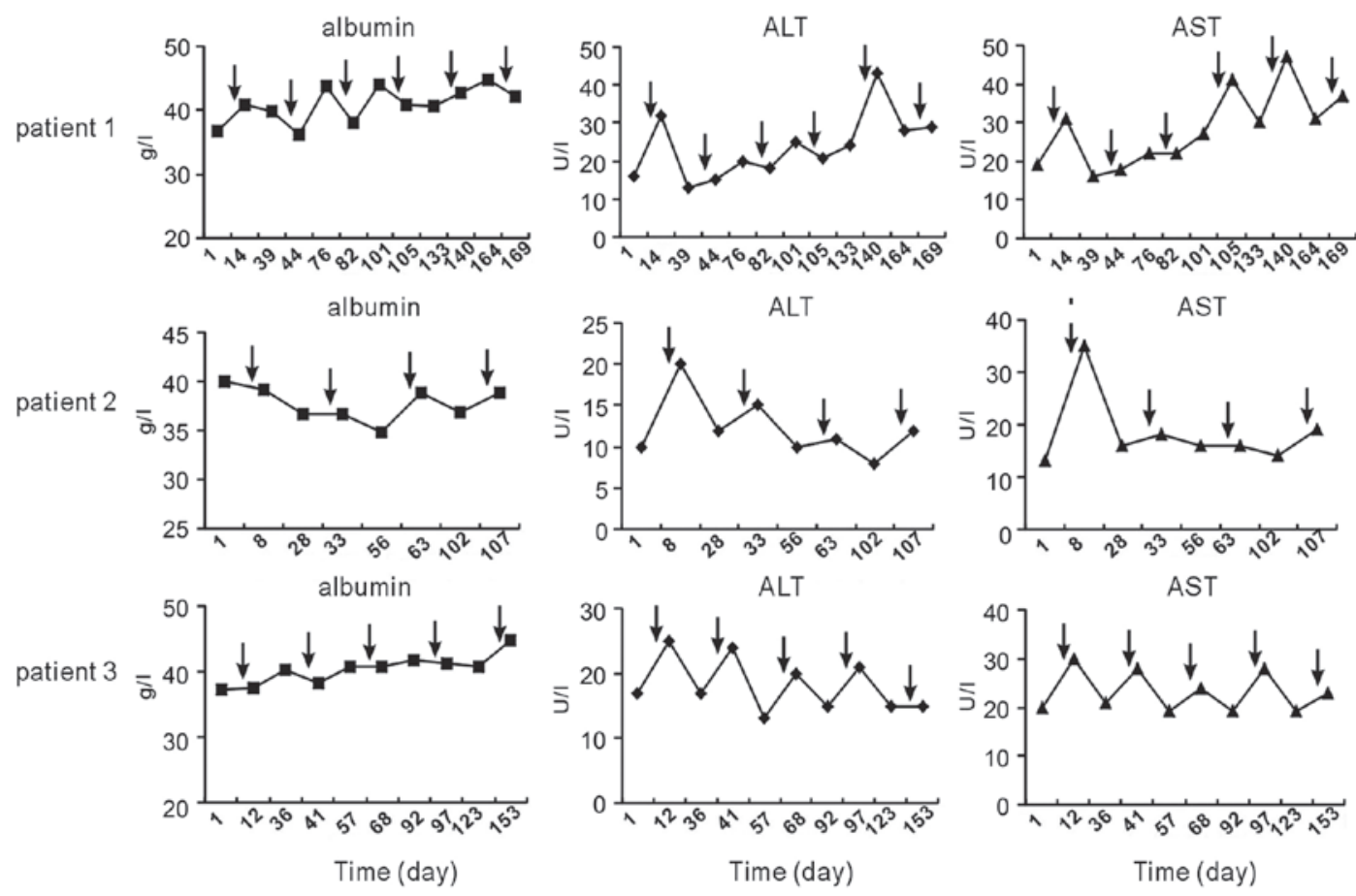

Figure 3. Liver-associated parameters before and after TP chemotherapy. Arrows indicate time-points of TP chemotherapy. Normal range: albumin, 40.0-55.0 g/1; ALT <50 U/1; AST <40 U/1. ALT, alanine transaminase; AST, aspartate aminotransferase; TP, paclitaxel and cisplatin.

\section{Discussion}

The present study reported on three patients with cancer who developed HTG following TP chemotherapy. No patient had any history of or any pre-existing lipid abnormalities, and exhibited no other predisposing factors, including chronic alcoholism, diabetes mellitus or hypothyroidism. Typically, HTG occurs in conjunction with low HDL levels and atherogenic small dense LDL particles (20). However, the present study found that the levels of physiologically important plasma 

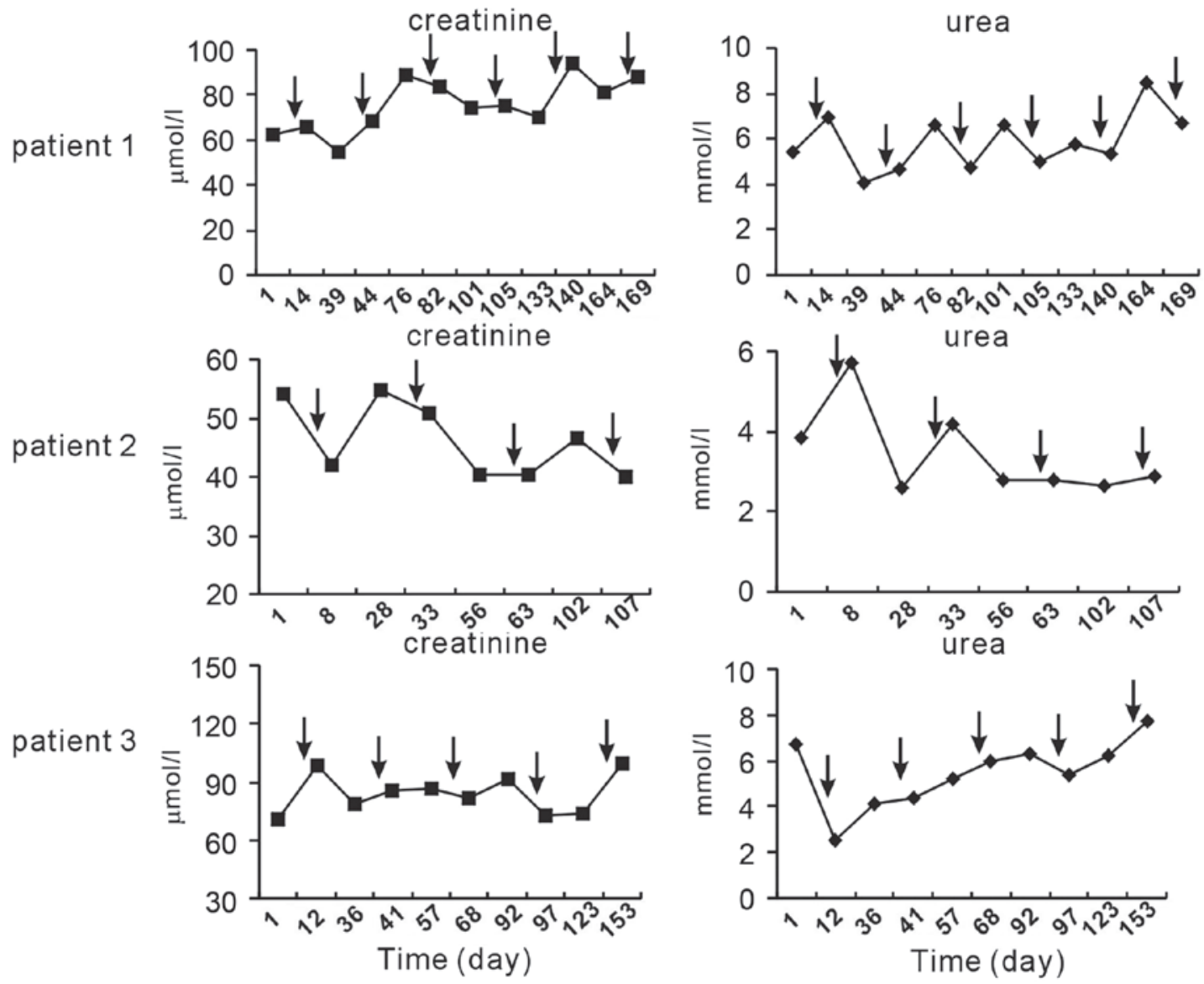

Figure 4. Serum levels of parameters associated with renal function before and after TP chemotherapy. Normal range: creatinine, $53.0-140.0 \mu \mathrm{mol} / 1$; urea, 2.40-7.20 mmol/l. Arrows indicate time-points of TP chemotherapy. TP, paclitaxel and cisplatin.
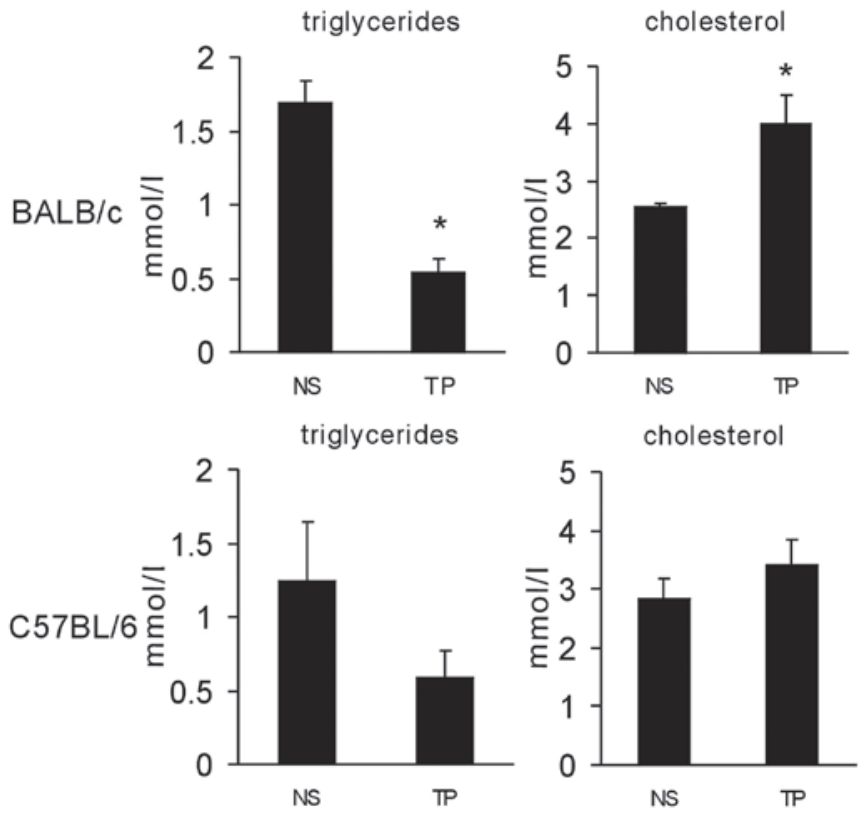

Figure 5. Chemotherapy with TP disturbed serum lipids in experimental animals. BALB/c mice were treated intravenously with paclitaxel $(10 \mathrm{mg} / \mathrm{kg}$ body weight) at day 1 , and cisplatin ( $5 \mathrm{mg} / \mathrm{kg}$ body weight) at days 1,2 and 3. C57BL/6 mice were administered intraperitoneally with the same dose of chemotherapeutical drugs. Serum was obtained on day 4 to evaluate the triglyceride and cholesterol levels. " $\mathrm{P}<0.05$ vs. NS. TP, paclitaxel and cisplatin. Results were expressed as the mean \pm standard deviation. NS, normal saline. cholesterol, LDL and HDL were normal or only minimally changed. The reason for the observed TG elevation remains elusive. In the three patients, it was observed that ALT and/or AST were transiently elevated after TP chemotherapy and showed a similar trend to serum $\mathrm{TG}$, while remaining in the normal range. Therefore, the fluctuation of TG may have resulted from impaired hepatic cellular function.

The significance of HTG in patients undergoing chemotherapy has remained elusive. In a clinical trial reported by Blumenschein et al (21) HTG had a prognostic value for bexarotene-treated patients with non-small cell lung cancer. Furthermore, the occurrence of high-grade HTG was highly correlated with increased survival (21). Of note, a marked elevation of TG appeared to be causally linked to acute pancreatitis. Acute pancreatitis induced by HTG usually occurs when serum TG levels exceed $20 \mathrm{mmol} / 1(16,22)$. A TG serum level of $>1,000 \mathrm{mg} / \mathrm{dl}$ can be used to determine the required therapy (23). Failure to consider lipid levels as the cause of disease can lead to clinical deterioration (24). In the present study, the serum TG levels in most patients who received chemotherapy was $<5 \mathrm{mmol} / \mathrm{l}$. However, there is evidence that serum TG levels as low as $343 \mathrm{mg} / \mathrm{dl}(3.9 \mathrm{mmol} / \mathrm{l})$ were able to trigger pancreatitis (25). Therefore, clinicians must be vigilant of acute pancreatitis in all patients who receive TP therapy. As HTG can impair the function of endothelial cells, it is necessary to protect the endothelium when it is encountered during treatment with TP chemotherapy. 
Notably, the present study observed an elevation of TG in patients following TP chemotherapy, whereas the opposite phenomenon was observed in mice. To mimic the clinical settings, mice were administered paclitaxel at day 1 and cisplatin at days 1,2 and 3. On day 4, serum TG were reduced in the mice, while cholesterol was increased. This difference from the observations in human patients may have resulted from different species or different dosage of chemotherapeutic agents.

In conclusion, the present study highlighted the requirement for clinicians to consider severe dyslipidemia as a possible side effect prior to initiating TP chemotherapy, which may result in complications.

\section{Acknowledgements}

The present study was supported by the National Natural Science Foundation of China (no. 81501609), the National Natural Science Foundation of China (no. 81301980), and the Chinese Postdoctoral Science Foundation (no. 2015M582553).

\section{References}

1. Fearon KC, Glass DJ and Guttridge DC: Cancer cachexia: Mediators, signaling, and metabolic pathways. Cell Metab 16 153-166, 2012.

2. Landen CN Jr, Birrer MJ and Sood AK: Early events in the pathogenesis of epithelial ovarian cancer. J Clin Oncol 26: 995-1005, 2008.

3. Nieman KM, Kenny HA, Penicka CV, Ladanyi A, Buell-Gutbrod R, Zillhardt MR, Romero IL, Carey MS, Mills GB, Hotamisligil GS, et al: Adipocytes promote ovarian cancer metastasis and provide energy for rapid tumor growth. Nat Med 17: 1498-1503, 2011.

4. Zhang Y, Daquinag AC, Amaya-Manzanares F, Sirin O, Tseng C and Kolonin MG: Stromal progenitor cells from endogenous adipose tissue contribute to pericytes and adipocytes that populate the tumor microenvironment. Cancer Res 72: 5198-5208, 2012.

5. Dirat B, Bochet L, Dabek M, Daviaud D, Dauvillier S, Majed B, Wang YY,Meulle A, Salles B,Le Gonidec S, et al: Cancer-associated adipocytes exhibit an activated phenotype and contribute to breast cancer invasion. Cancer Res 71: 2455-2465, 2011.

6. Michalek RD, Gerriets VA, Jacobs SR, Macintyre AN, MacIver NJ, Mason EF, Sullivan SA, Nichols AG and Rathmell JC: Cutting edge: Distinct glycolytic and lipid oxidative metabolic programs are essential for effector and regulatory CD4+ T cell subsets. J Immunol 186: 3299-3303, 2011.

7. Huang SC, Everts B, Ivanova Y, O'Sullivan D, Nascimento M, Smith AM, Beatty W, Love-Gregory L, Lam WY, O'Neill CM, et al: Cell-intrinsic lysosomal lipolysis is essential for alternative activation of macrophages. Nat Immunol 15: 846-855, 2014.

8. Pencheva N, Tran H, Buss C, Huh D, Drobnjak M, Busam K and Tavazoie SF: Convergent multi-miRNA targeting of ApoE drives LRP1/LRP8-dependent melanoma metastasis and angiogenesis. Cell 151: 1068-1082, 2012.
9. Fang L, Choi SH, Baek JS, Liu C, Almazan F, Ulrich F, Wiesner P, Taleb A, Deer E, Pattison J, et al: Control of angiogenesis by AIBP-mediated cholesterol efflux. Nature 498: $118-122,2013$.

10. Ito Y, Azrolan N, O'Connell A, Walsh A and Breslow JL: Hypertriglyceridemia as a result of human apo CIII gene expression in transgenic mice. Science 249: 790-793, 1990.

11. Hsia SH, Connelly PW and Hegele RA: Successful outcome in severe pregnancy-associated hyperlipemia: A case report and literature review. Am J Med Sci 309: 213-218, 1995.

12. Calza L, Manfredi R and Chiodo F: Dyslipidaemia associated with antiretroviral therapy in HIV-infected patients. J Antimicrob Chemother 53: 10-14, 2004

13. Austin MA: Plasma triglyceride and coronary heart disease. Arterioscler Thromb 11: 2-14, 1991.

14. Reaven GM: Banting lecture 1988. Role of insulin resistance in human disease. Diabetes 37: 1595-1607, 1988.

15. Maher NG and Ramaswamykanive H: Use of plasmapheresis in managing the diagnostic dilemma of symptomatic hypertriglyceridemia. Case Rep Gastrointest Med 2012: 501373, 2012.

16. Kyriakidis AV, Raitsiou B, Sakagianni A, Harisopoulou V, Pyrgioti M, Panagopoulou A, Vasilakis N and Lambropoulos S: Management of acute severe hyperlipidemic pancreatitis. Digestion 73: 259-264, 2006.

17. Athyros VG, Giouleme OI, Nikolaidis NL, Vasiliadis TV, Bouloukos VI, Kontopoulos AG and Eugenidis NP: Long-term follow-up of patients with acute hypertriglyceridemia-induced pancreatitis. J Clin Gastroenterol 34: 472-475, 2002.

18. Yadav D and Pitchumoni CS: Issues in hyperlipidemic pancreatitis. J Clin Gastroenterol 36: 54-62, 2003.

19. Bollen TL, van Santvoort HC, Besselink MG, van Leeuwen MS, Horvath KD, Freeny PC and Gooszen HG; Dutch Acute Pancreatitis Study Group: The Atlanta Classification of acute pancreatitis revisited. Br J Surg 95: 6-21, 2008.

20. Subramanian S and Chait A: Hypertriglyceridemia secondary to obesity and diabetes. Biochim Biophys Acta 1821: 819-825, 2012.

21. Blumenschein GR Jr, Khuri FR, von Pawel J, Gatzemeier U, Miller WH Jr, Jotte RM, Le Treut J, Sun SL, Zhang JK, Dziewanowska ZE and Negro-Vilar A: Phase III trial comparing carboplatin, paclitaxel, and bexarotene with carboplatin and paclitaxel in chemotherapy-naive patients with advanced or metastatic non-small-cell lung cancer: SPIRIT II. J Clin Oncol 26: 1879-1885, 2008.

22. Gan SI, Edwards AL, Symonds CJ and Beck PL: Hypertriglyceridemia-induced pancreatitis. A case-based review. World J Gastroenterol 12: 7197-7202, 2006.

23. Jain P, Rai RR, Udawat H, Nijhawan S and Mathur A: Insulin and heparin in treatment of hypertriglyceridemia-induced pancreatitis. World J Gastroenterol 13: 2642-2643, 2007.

24. Markota A, Knehtl M, Sinkovic A, Ekart R, Hojs R and Bevc S: Plasma exchange treatment for acute hyperlipidemic pancreatitis with falsely low levels of serum triglycerides- a case report. Transfus Apher Sci 51: 178-180, 2014.

25. Monib SM and El-Barbary HM: Acute relapsing pancreatitis with pseudocyst formation due to sporadic hypertriglyceridemic pancreatitis: A case report. Indian J Surg 75 (Suppl 1): S340-S344, 2013. 